\title{
Myopericarditis diagnosed by a 64-slice coronary CT angiography "triple rule out" protocol
}

\author{
Kevin M. Takakuwa $\cdot$ Bon S. Ku $\cdot$ Ethan J. Halpern
}

Received: 6 April 2010 / Accepted: 15 June 2010/Published online: 21 August 2010

(C) Springer-Verlag London Ltd 2010

\begin{abstract}
We report a case of myopericarditis in a 30-yearold male complaining of shortness of breath. In an emergency department (ED) setting, the symptoms of myopericarditis may overlap with many disease entities and can be a challenging diagnosis to make. However, with the use of a 64-section coronary CT angiography in a "triple rule out" (TRO) protocol, we were able to detect a large pericardial effusion surrounding the heart and moderate global hypokinesis in the setting of normal-sized heart chambers and normal coronary arteries. We were further able to exclude pulmonary embolism and thoracic dissection. This is the first reported case of diagnosing myopericarditis using a TRO protocol. It demonstrates the usefulness of TRO in making an emergent diagnosis of myopericarditis while excluding other lifethreatening diseases that can lead to earlier appropriate ED disposition and care.
\end{abstract}

Keywords Myopericarditis · Myocarditis · Pericarditis · $\mathrm{CT}$ angiography $\cdot$ Sixty-four section $\cdot$ Triple rule-out

\section{Introduction}

The historically accepted gold standard for diagnosing myocarditis is endomyocardial biopsy [1]. With advances

\author{
K. M. Takakuwa $(\bowtie) \cdot$ B. S. Ku \\ Department of Emergency Medicine, \\ Thomas Jefferson University Hospital, \\ 1020 Sansom Street, Suite 239 Thompson Building, \\ Philadelphia, PA 19107, USA \\ e-mail: kevin.takakuwa@jefferson.edu \\ E. J. Halpern \\ Department of Radiology, Thomas Jefferson University Hospital, \\ 111 South 11th St, \\ Philadelphia, PA 19107, USA
}

in technology, the diagnosis of myocarditis is being aided by MRI [1,2] and echocardiography [3]. Cardiac MRI is not generally available for ED patients. Although echocardiography is frequently used to evaluate for pericardial effusion and cardiac wall motion abnormality in the ED patient, echocardiography cannot exclude coronary disease, pulmonary embolism or aortic dissection as causes of chest pain. Both myocarditis and pericarditis are most often offered as alternative diagnoses after these other lifethreatening disease processes have been ruled out. The ED workup to exclude these other diagnoses can be lengthy, expensive and ultimately inconclusive. We report on an atypical case presentation of undifferentiated shortness of breath that was rapidly diagnosed as myopericarditis in the ED by a single test: a 64-section coronary CT angiography in a "triple rule-out" (TRO) protocol.

\section{Report}

A 30-year-old man with no previous medical problems presented to the ED complaining of the sudden onset of shortness of breath. His symptoms had started $2 \mathrm{~h}$ prior to arrival and were associated with chest pain, back pain and a cough. The chest pain was described as substernal, sharp, tearing and radiating to his mid-back. He had associated diaphoresis, palpitations, dizziness and subjective fevers. His symptoms started at rest and worsened with deep breaths. He took ibuprofen at home with some relief of symptoms. Vital signs at presentation were temperature $36.7 \mathrm{C}(98.1 \mathrm{~F})$, blood pressure $145 / 100$, pulse rate 166 beats/min, respiratory rate 28 breaths/min, and pulse oximetry $100 \%$ on room air. The patient appeared anxious and had an unremarkable physical examination with the exception of his heart rate. His heart was tachycardiac but 


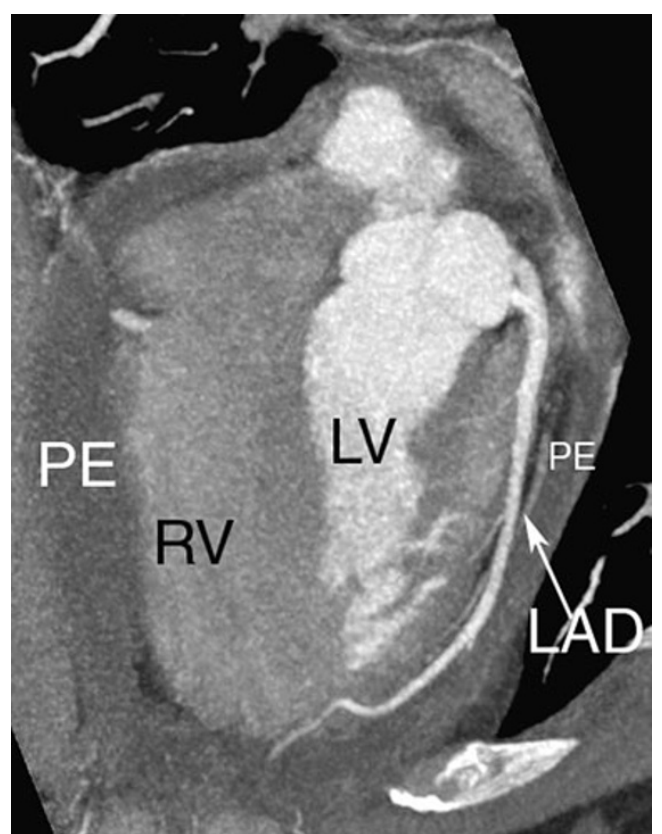

Fig. 1 Four chamber view. TRO study demonstrating mild-tomoderate pericardial effusion surrounding the heart. PE - pleural effusion, RV - right ventricle, LV - left ventricle, LAD - left anterior descending coronary artery

regular at a rate in the $150 \mathrm{~s}$. He had normal S1 and S2 without any extra heart sounds, murmurs, gallops, or rubs.

Laboratory values were WBC count $12,600 / \mathrm{mm}^{3}$, hemoglobin $16.4 \mathrm{~g} / \mathrm{dl}$, hematocrit level $46.7 \%$, platelets 223,000 / $\mathrm{mm}^{3}$, sodium $138 \mathrm{mEq} / 1$, potassium $5.2 \mathrm{mEq} / 1$, chloride $104 \mathrm{mEq} / \mathrm{l}$, carbon dioxide $24 \mathrm{mEq} / \mathrm{l}$, blood urea nitrogen $18 \mathrm{mg} / \mathrm{dl}$, creatinine level $1.2 \mathrm{mg} / \mathrm{dl}$, glucose concentration

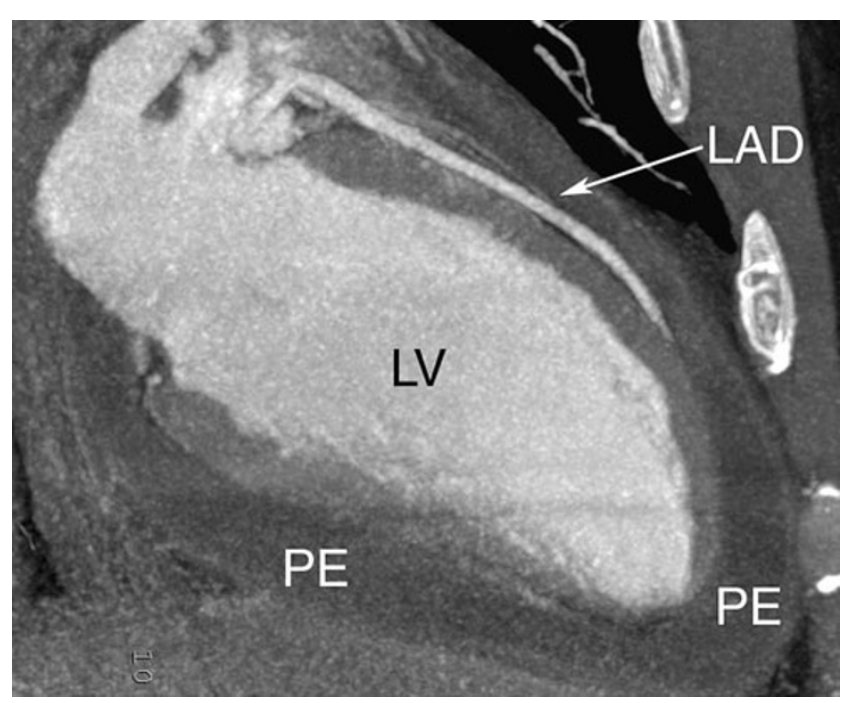

Fig. 2 Two chamber view. TRO study demonstrating mild-tomoderate pericardial effusion surrounding the heart. PE - pleural effusion, LV - left ventricle, LAD - left anterior descending coronary artery

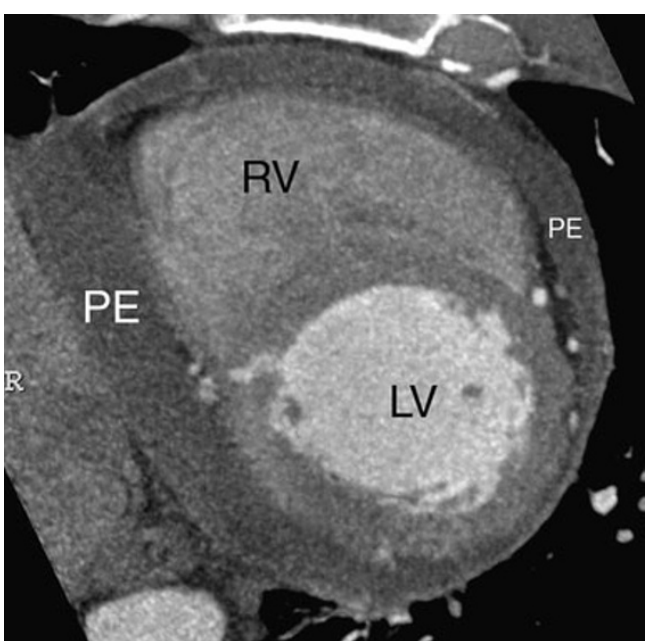

Fig. 3 Short axis view. TRO study demonstrating mild-to-moderate pericardial effusion surrounding the heart. PE - pleural effusion, RV right ventricle, $L V$ - left ventricle

$113 \mathrm{mg} / \mathrm{dl}$, myoglobin $13 \mathrm{ng} / \mathrm{ml}$, and troponin $<0.05 \mathrm{ng} / \mathrm{ml}$. Chest radiography revealed no abnormalities. An initial electrocardiogram showed a rate of $151 \mathrm{bpm}$ with no significant ST-T wave abnormalities noted. A TRO study was performed, which demonstrated a mild-to-moderate pericardial effusion surrounding the heart (Figs. 1, 2, 3 and 4) and moderate global hypokinesis with an ejection fraction of $35 \%$. He had normal-sized heart chambers and normal coronary arteries (left anterior descending and right coronary artery shown). The aorta and pulmonary arteries were entirely normal. Given the clinical scenario and TRO findings, the patient was admitted to the hospital with a tentative diagnosis of myopericarditis. The patient had a

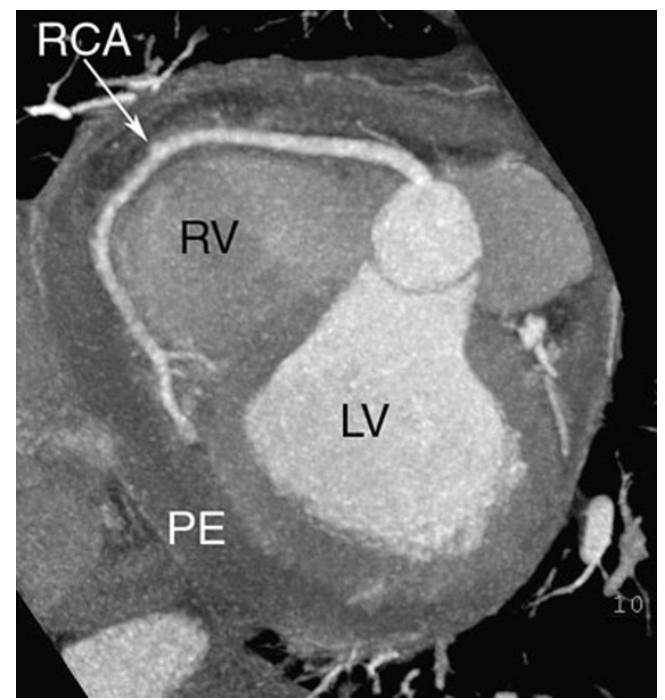

Fig. 4 Left anterior oblique view optimized to visualize the full length of the RCA (right coronary artery). TRO study demonstrating mild-to-moderate pericardial effusion surrounding the heart. PE pleural effusion, RV - right ventricle, LV - left ventricle 
subsequent transthoracic echocardiogram that demonstrated a small-to-moderate pericardial effusion without evidence of tamponade and mild global left ventricular dysfunction, confirming the diagnosis made by the TRO study.

Based on the findings, the patient was managed with NSAIDS and aspirin. He had a gradual improvement in his symptoms, and his ejection fraction improved to 55-60\% by his third day of hospitalization. By the time of his hospital discharge on day 6 , his exam revealed a pericardial friction rub. The patient was discharged with no further sequelae.

\section{Discussion}

Myopericarditis, or inflammation of the myocardium and pericardium, can present with an overlap of the symptoms of myocarditis (flu-like symptoms such as fever, fatigue and myalgias) and pericarditis (sharp or pleuritic chest pain that is relieved with sitting forward and worsened by laying back) $[4,5]$. In this case, our patient had symptoms of both: subjective fevers and diaphoresis with substernal chest pain. He additionally had clinical complaints that could be consistent with a thoracic dissection given the tearing pain radiating to his mid-back or a pulmonary embolism with his shortness of breath, tachycardia and pleuritic chest pain. Given his presentation and chief complaint of shortness of breath, the ED physician decided to order the TRO as the first diagnostic test. A diagnosis for myopericarditis was made within $2 \mathrm{~h}$ from ED arrival time.

Our rapid ED diagnosis with the TRO protocol allowed us to admit the patient immediately and initiate treatment while avoiding other lengthier testing. In the absence of a TRO study, ruling out pulmonary embolism and thoracic dissection would have required a ventilation-perfusion scan and chest CT (assuming that the chest CT was not performed first that would have revealed the pericardial effusion). The ECG was not helpful as it did not reveal characteristic pericarditis related stage 1 ST-segment elevations or PR depressions, stage 2 T-wave flattening or stage 3 deep T-wave inversions. The ECG also did not appear to look like an acute myocardial infarction, which must be considered in the differential diagnosis [6, 7]. The unexplained tachycardia may have been a clue to myocarditis, though tachycardia is non-specific and can also be seen with a pulmonary embolism. It is interesting to note that the patient did not develop a pericardial friction rub until his 3rd day of hospitalization. Presumably, the large volume of pericardial effusion masked the presence of the friction rub at the initial presentation.

We know of one reported case of using 64-multi-slice CT to exclude coronary artery disease in a patient who was already diagnosed with myopericarditis based on the clinical picture [8]. The CT was performed 2 days into hospitalization and did not reveal any findings that would have helped make the diagnosis. Our case is unique in that we actually made the diagnosis using our TRO protocol while excluding other life-threatening diseases. Another report on two cases of acute myocarditis also described CT findings after admission to the hospital [9]. Both of their patients had ST segment ECG abnormalities and abnormal cardiac enzymes.

We believe this case illustrates the value of the 64section TRO in the ED setting to help rapidly distinguish emergent, life-threatening illnesses from more benign disease processes [10]. In this case, we were able to initiate the appropriate medical treatment and not initiate alternative treatments like thrombolysis, appropriate treatment for an acute myocardial infarction but that may be contraindicated in myopericarditis.

\section{References}

1. Magnani JW, Dec GW (2006) Myocarditis: current trends in diagnosis and treatment. Circulation 113:876-890

2. Abdel-Aty H, Boye P, Zagrosec A et al (2005) Diagnostic performance of cardiovascular magnetic resonance in patients with suspected acute myocarditis. J Am Coll Cardiol 45:1815-1822

3. Skouri HN, Dec GW, Friedrich MG, Cooper LT (2006) Noninvasive imaging in myocarditis. J Am Coll Cardiol 48:2085-2093

4. Pawsat DE, Lee JY (1998) Inflammatory disorders of the heart. Pericarditis, myocarditis, and endocarditis. Emerg Med Clin N Am 16:665-681

5. Brady WJ, Ferguson JD, Ullman EA, Perron AD (2004) Myocarditis: emergency department recognition and management. Emerg Med Clin N Am 22:865-885

6. Dec GW, Waldman H, Southern J, Fallon JT, Hutter AM Jr, Palacios I (1992) Viral myocarditis mimicking acute myocardial infarction. J Am Coll Cardiol 20:85-89

7. Miklozek CL, Crumpacker CS, Royal HD, Come PC, Sullivan JL, Abelmann WH (1988) Myocarditis presenting as acute myocardial infarction. Am Heart J 115:768-776

8. Shturman A, Chernihovski A, Goldfeld M, Furer A, Wishniak A, Roguin N (2007) Usefulness of 64 multi-slice computed tomography in acute myopericarditis. IMAJ 9:333-334

9. Brooks MA, Sane DC (2007) CT findings in acute myocarditis: two cases. J Thorac Imaging 22:277-279

10. Takakuwa KM, Halpern EJ (2008) Evaluation of a "triple ruleout" coronary CT angiography protocol: use of 64-section CT in low-to-moderate risk emergency department patients suspected of having acute coronary syndrome. Radiology 248:438-446 